\title{
DESIGN OF COUPLED SLIDER CRANK MECHANISM FOR ORBITING MOTION
}

\author{
Veg, E. ; Veg, A. ; Sinikovic, G. ; Andrejevic, R. ${ }^{*} \&$ Gubeljak, N. ${ }^{* *}$ \\ ${ }^{*}$ University of Belgrade, Faculty of Mech. Engineering, Kraljice Marije 16, 1100 Belgrade, Serbia \\ ** University of Maribor, Faculty of Mechanical Engineering, Smetanova 17, 2000 Maribor, Slovenia \\ E-Mail: eveg@mas.bg.ac.rs, aveg@mas.bg.ac.rs, gsinikovic@mas.bg.ac.rs, randrejevic@mas.bg.ac.rs, \\ nenad.gubeljak@um.si
}

\begin{abstract}
The paper describes development of a novel orbiting mechanism. Target design is a shaking mechanism with a planar figure eight closed loop. Shaking dynamics depends on a cycling frequency as well on a reciprocating displacement. These two parameters are in the focus of preliminary design and analysis. Computational optimization and path definition were interrelated to core engineering work. Crucial novelty is an orbital motion created by a coupled slider crank mechanism. An academic package of the Solid Works Motion 2010 was employed for parts and assembly design, as well as for the subsequent mechanism analysis. The outcome design proved itself in operation with a smooth shaking motion over a closed loop and even more the uniformly distributed acceleration peaks within each cycle.

(Received in January 2014, accepted in January 2015. This paper was with the authors 5 months for 3 revisions.)
\end{abstract}

Key Words: Orbiting Mechanism, Shaking Function, Fluid Stirring, Simulated Motion

\section{INTRODUCTION}

Chemical inactivation [1] is usually performed in a formaldehyde solution by orbiting mechanism. During the inactivation period the whole content must be carefully stirred or shaken in order to prevent stratification of either viruses or the supplement agents in the composition. Available for this purpose are the magnetic stirrers, vortex homogenizers, rotators, shakers, mixers and similar devices. There is a significant difference between mixing, stirring and shaking [2], mainly in the aspect of fluid mechanics, combined with bio chemical effects. None of the available devices can provide a smooth, foamless stirring or shaking. Unlike shaking, stirring usually creates a central vortex, whirling the suspension in an uneven fashion: at the central whirling axis the motion is very intensive while the peripheral points remain less agitated. Shaking is the preferred choice for the process of viral inactivation, regarding the controlled mixing intensity and evenly distributed field of velocities and accelerations in the whole content. Among the existing shaking devices [3], shown in Fig. 1, there is not a single one that completely fulfils demand for a controllably smooth, but intensive shaking. Therefore, the main target of the foreseen engineering work was to develop an appropriate and efficient shaking device aimed for vaccine production. The novel design of a coupled crank mechanism for orbiting motion truly satisfies the established criteria for effective shaking.

It follows a brief review of shaking functions with conventional devices:

- Reciprocating is a simple forth and back motion, suffering from intensive jerk at the points of sense shifting.

- Rocking is a rather monotonous motion.

- Circular vibration keeps the content of the vertically placed bottles almost steady.

- Waving is a 3D motion. Actually it is a coupled rocking around two orthogonal axes (x,y).

- Simple orbiting is a relatively steady motion, similar to rocking.

- Over heading is an unacceptable motion for the foreseen inactivation process. 


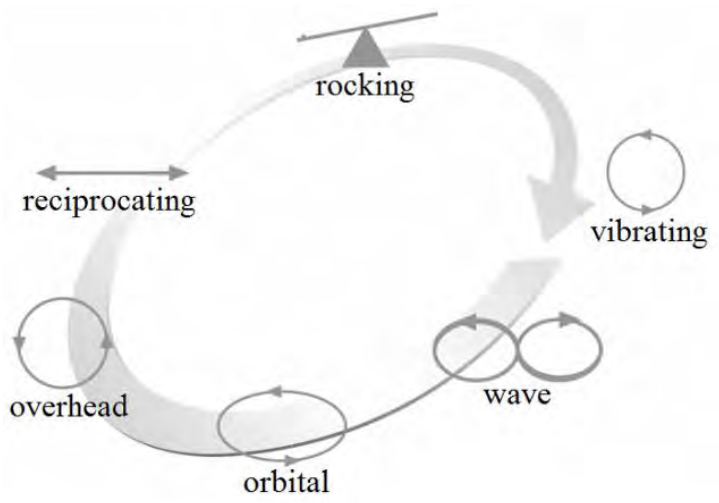

Figure 1: Conventional shaking paths.

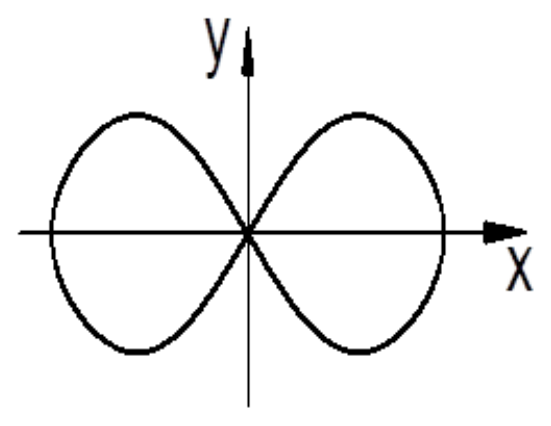

Figure 2: Planar Figure Eight Orbiting Path (PFEOP).

\section{PLANAR FIGURE EIGHT ORBITING PATH (PFEOP)}

Unlike the conventional shaking functions it was worth investigating a novel orbiting motion in a form of planar figure eight (Fig. 2). Prior to any further design activity a true computational simulation is undertaken. Benefits of innovative shaking motion over planar eight orbiting path is very persuasive, such as:

- the complete loop is closed (no interrupts, no dwells),

- travelling over such a path is smooth (jerk-free),

- the sense of motion is gradually altered along the path,

- the size, shape and the cycling frequency on the pattern are adjustable.

\section{HOW TO GENERATE "PFEOP"}

Conventional approach to this issue would be to find a conventional PFEOP generating device and to adapt it to a particular application. In that sense the most common and popular arbitrary path generator is a motorized coordinate table as shown in Fig. 3.

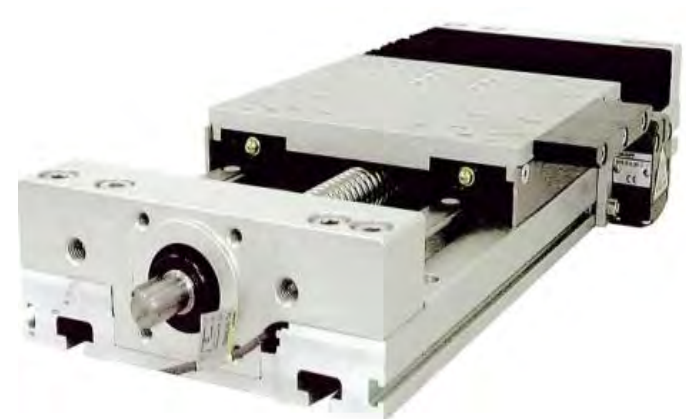

Figure 3: Motorized coordinate table.

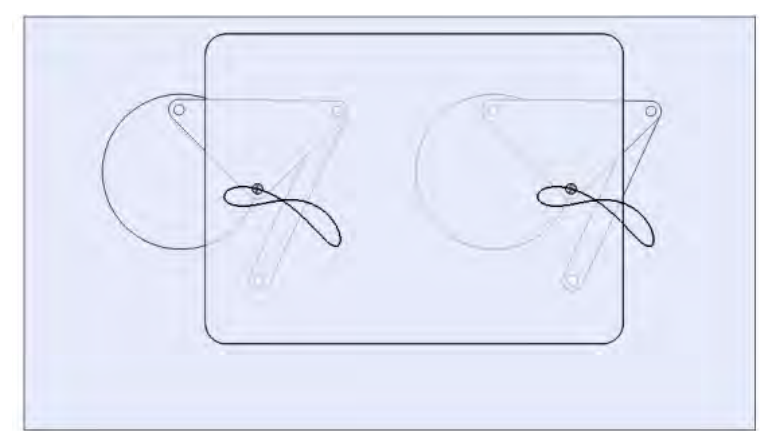

Figure 4: Dual four bar linkage as a generator of PFEOP.

The advantages of such a unit are numerous [4] (e.g. programmable path shape, its size and motion dynamics, high loading capacity, market availability, etc.). On the contrary, its disadvantages are few but serious. Since the linear motion along each axis is relatively short, a shift in direction occurs sharply, leading to an intensive wear of the drive unit. The inertial forces on such snap moves are intensive and out of balance [5].

The other conventional solution for PFEOP generation would be a customized four bar linkage [6]. Coupled configuration of two identical four bar linkages (Fig. 4) translates a platform over PFEOP. The mechanism is rather complex, while the generated pattern is significantly distorted. Also, the acceleration peaks are extremely abrupt. Modification of such a shaking mechanism is quite complicated. 
Neither of the aforementioned options appeared to be an acceptable solution. Instead, a new concept of PFEOP generating mechanism was conceived [7, 8]. Here follows the explanation of the initial idea. The clue to the invention was a coupling of two crank mechanisms, driven by electric motor (Fig. 5) [9].

While the crank mechanism no. 1 (CM1) completes half of a cycle (forth piston motion 15), the crank mechanism no. 2 (CM2) placed on the CM1 piston, travels a whole cycle. Outcome of these coupled motions depicts half of a figure eight path. When completed one CM1 forth and back full cycle of CM1 and two complete CM2 cycles, the required figure eight path is encircled [10].

Motion of the CM2 piston, which is now the executive of the integrated Coupled Crank Mechanism (CCM) can be described as:

- position along the $\mathrm{x}$-axis (CM1 piston motion) $x_{A}=R_{1} \sin (\omega t)$

- position along the y-axis (CM2 piston motion) $y_{A}=R_{2} \sin (2 \omega t+\Phi)$

$$
\begin{array}{ll}
x, y & - \text { Cartesian coordinates, } \\
R_{1}, R_{2} & \text { - respective radii of CM1 and CM2, } \\
\omega, 2 \omega & \text { - respective angular speeds of CM1 and CM2, } \\
\Phi & \text { - phase lag between CM1 and CM2. }
\end{array}
$$

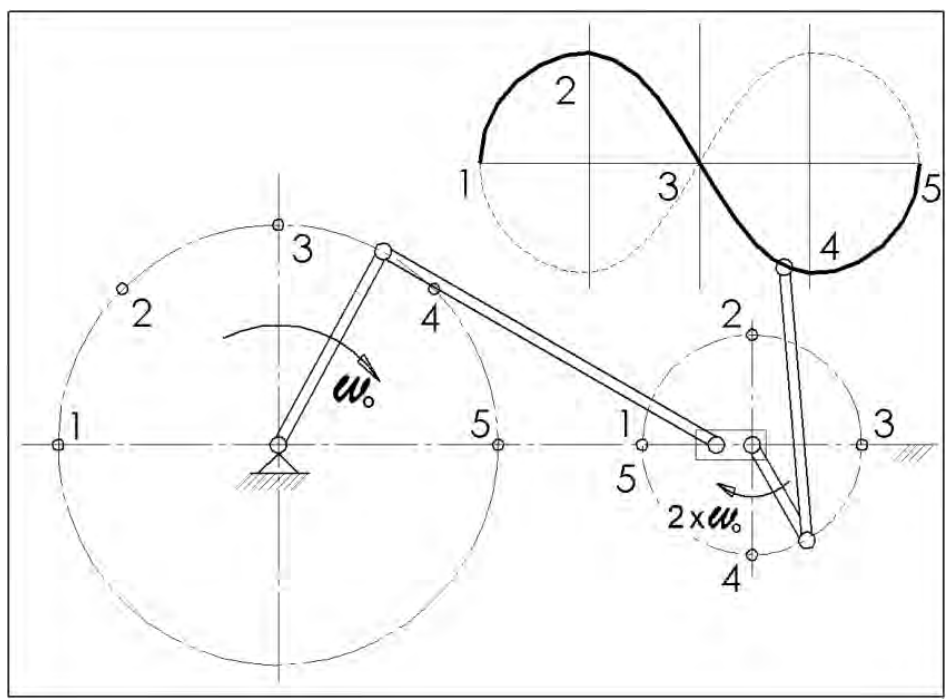

Figure 5: Path composition of two slider crank motions (1/2 cycle of a PFEOF).

Figure eight orbiting path is generated when the ratio between CM1 and CM2 angular speed is ultimately:

$$
\omega_{\mathrm{CM} 1}: \omega_{\mathrm{CM} 2}=1: 2
$$

Fig. 5 illustrates step-by-step half of a PFEOF generation. Since the motion ratio between $\omega_{C M 1}$ and $\omega_{C M 2}$ is $1: 2$, while the Crank1 changes for angle $\theta$, the Crank2 moves over $2 \theta$. This planar motion occurs in the XY plane. The dual crank mechanism, driven both by the CM1 and CM2, due to (1), (2), (3) and (4) considering that:

$$
\omega_{\mathrm{CM} 1} t=\theta \text { and } \omega_{\mathrm{CM} 2} t=2 \theta
$$

will travel over $\mathrm{X}$ and $\mathrm{Y}$ coordinates in absolute coordinate system, Table I.

Table I: Crank angles vs. corresponding normalized absolute coordinates.

\begin{tabular}{|c|c|c|c|c|c|c|c|c|c|c|}
\hline$\theta\left[^{\circ}\right]$ & 0 & 10 & 20 & 30 & 40 & 50 & 60 & 70 & 80 & 90 \\
\hline$X$ & 0.00 & 0.17 & 0.34 & 0.50 & 0.64 & 0.77 & 0.87 & 0.94 & 0.98 & 1.00 \\
\hline$Y$ & 0.00 & 0.34 & 0.64 & 0.87 & 0.98 & 0.98 & 0.87 & 0.64 & 0.34 & 0.00 \\
\hline
\end{tabular}




\section{DIMENSIONAL AND DYNAMICAL OPTIMIZATION OF THE CCM}

By engaging the great simulating potential of CAD software, several CCM configurations were examined. The basic task was to harmonize PFEOP size and shape, as well as the travelling velocity over it, in order to optimize the map of accelerations.

Axiomatic contemplation of possible proportion in crank radii $\left(R_{1}\right.$ and $\left.R_{2}\right)$ of respectively CM1 and CM2 ultimately led to $2: 1$ rate, dictating a natural harmony of curvilinear motion. Obviously any other proportion $R_{1}$ to $R_{2}$ would disorder a smooth motion over PFEOP. Actually, twice the $R_{1}$ is the longitudinal span, while twice the $R_{2}$ is the transversal span.

Finally the overall range for $R_{1}$ and $R_{2}$ is determined to be $30 / 15 \mathrm{~mm}$, regarding the extremely large strokes at orbiting motion. Subsequently analysis revealed that even moderate values of radii (10,5 mm) provide sufficiently effective shaking: $R_{1}=30 \mathrm{~mm}, R_{2}=15 \mathrm{~mm}$.

The longitudinal axis of the PFEOP can also take a curvilinear form, depending on the phase lag $\Phi$ between CM1 and CM2 angular motion. The greater the difference in a phase lag, the closer the shape to a croissant form. Therefore the phase lag $\Phi$ is varied between $0^{\circ}$ and $40^{\circ}$, to check out the possible positive effects on the map of accelerations. As expected, besides the difference of the pattern shape, there was no significant influence on acceleration. Finally the angular speed $\omega$ is being varied within the range $5 \mathrm{~s}^{-1}$ through $15 \mathrm{~s}^{-1}$. Table II expresses the map of PFEOP accelerations for the prominent points (3-4-5), with the angular speed variation $\left(5 \mathrm{~s}^{-1}, 10 \mathrm{~s}^{-1}, 15 \mathrm{~s}^{-1}\right)$. Acceleration at the other symmetrical points is equal to the quoted ones by the amount and opposite by the sign. A more detailed overview of acceleration fluctuation is presented with the acceleration vs. time chart, where the prominent points on the path are clearly illustrated in Fig. 6.

Table II: Acceleration in prominent points.

\begin{tabular}{|c|c|c|c|}
\hline $\begin{array}{c}\text { Total acceleration } \\
{\left[\mathrm{m} / \mathrm{s}^{2}\right]}\end{array}$ & 5 & 10 & 15 \\
\hline$A_{3}$ & 0.644 & 2.289 & 5.902 \\
\hline$A_{4}$ & 1.721 & 7.007 & 15.509 \\
\hline$A_{5}$ & 0.102 & 0.777 & 1.357 \\
\hline
\end{tabular}

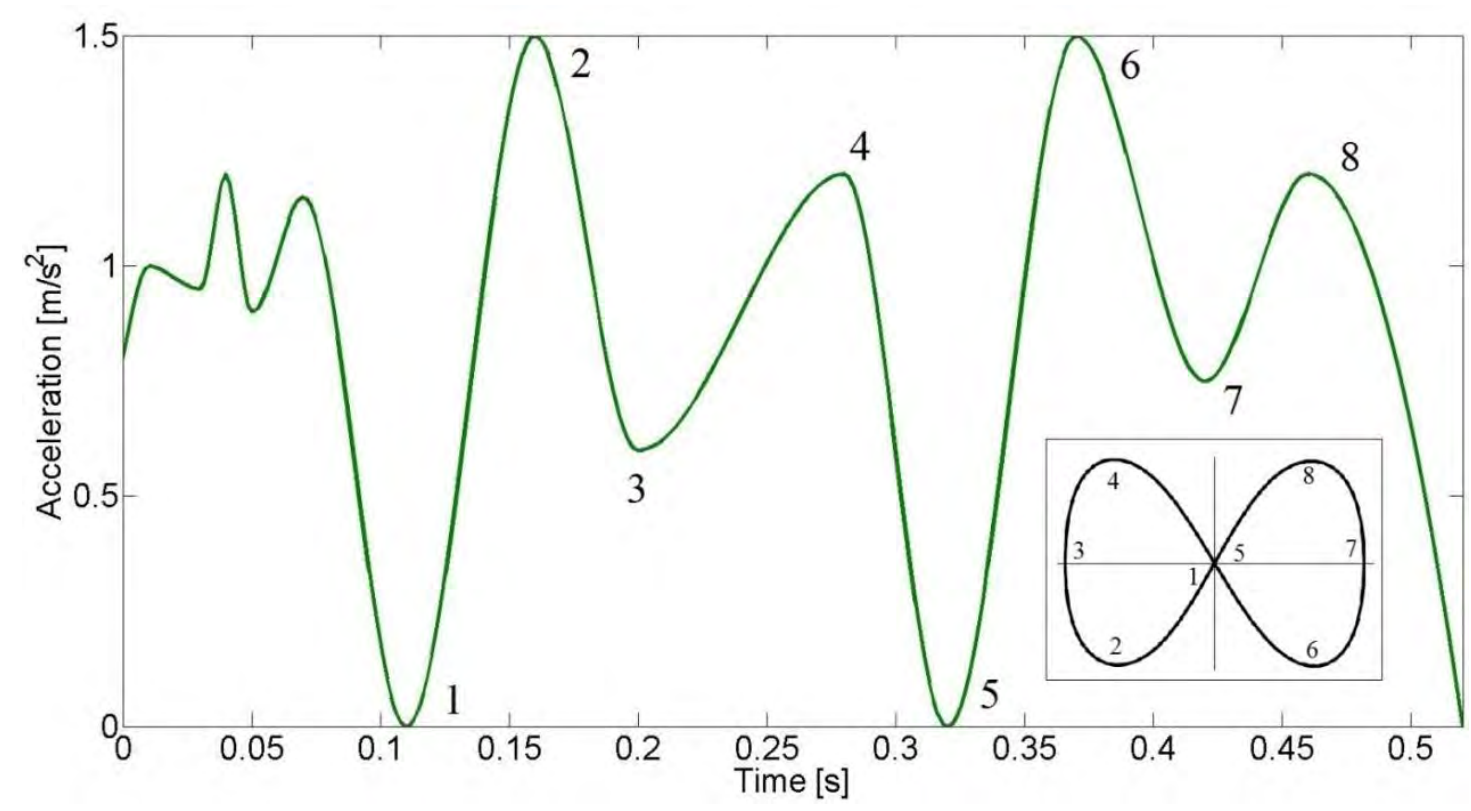

Figure 6: Acceleration vs. time $\omega=15 \mathrm{~s}^{-1}$, and prominent points of the path. 
Even a brief overview of the acceleration chart reveals a uniform distribution and density of its turning points. However in each orbiting cycle the acceleration slope inverts the sign eight times (at each prominent point). This event occurs more frequently than with any other similar device. Such a dynamical shaking motion or, rather, a constant shifting between positive and negative sloping, brings a great benefit in shaking.

On the other hand, the shaking intensity can be gradually tuned by the angular speed adjustment. Obviously a narrow range of angular speed variation $\left(5 \mathrm{~s}^{-1}\right.$ to $\left.15 \mathrm{~s}^{-1}\right)$ creates a considerable span in acceleration, approximately 1:10. This outstanding dynamical ability of the CCM justifies the soundness of the PFEOP intuitive selection.

\section{DYNAMIC MODEL OF A SLIDER-CRANK MECHANISM}

The crank performs pure rotation, the coupler performs planar motion, and the slider performs pure translation. Firstly the mechanism has to be dismantled into parts in order to create a dynamic model. It is necessary to define a model of each part, as well as the model of the whole mechanism.

\subsection{Dynamic model of the crank}

Equivalent dynamic model of the crank is formed by a decomposition of the overall crank mass $m_{2}$ into two masses: first $m_{20}$ fixed at the rotation point $\mathrm{O}$, and the second $m_{2 \mathrm{~A}}$, fixed at the joint A (Fig. 7).

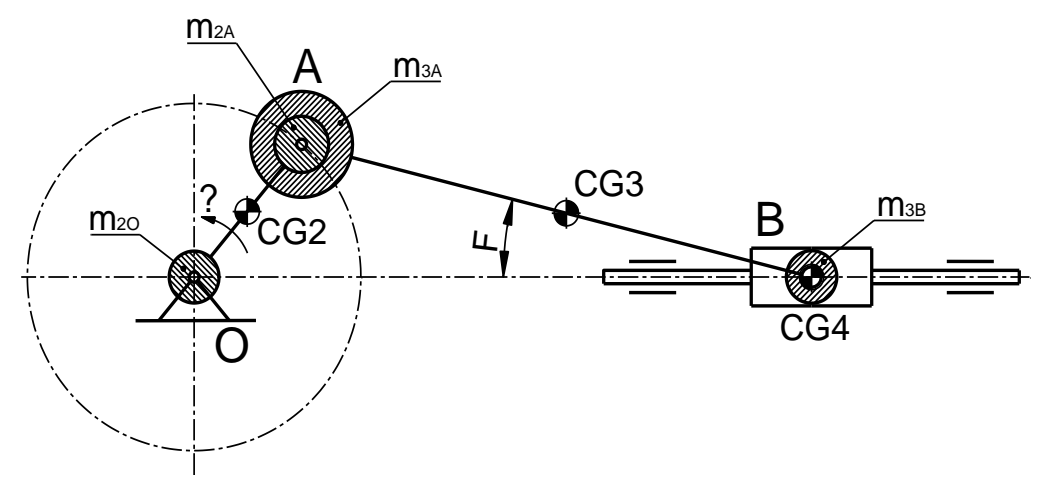

Figure 7: Dynamic model of the mechanism.

The equivalence between a real and a virtual crank must be perfect, particularly in the sense of unbalance and for that the following conditions must be fulfilled:

$$
\begin{gathered}
m_{2}=m_{2 \mathrm{O}}+m_{2 \mathrm{~A}} \\
m_{2} r_{G 2}=m_{2 \mathrm{~A}} r \\
m_{2 \mathrm{~A}}=m_{2} \frac{r_{G 2}}{r}
\end{gathered}
$$

\subsection{Dynamic model of the coupler}

Any member that performs planar motion with its mass $m$ and moment of inertia $I$ can be substituted with equivalent pair of concentrated masses. The inertia moment of these two virtual masses has to be equal to the inertia moment of the actual member $[4,11]$.

In order to determine the lump masses following conditions have to be fulfilled:

- Overall mass of the member is equal to the sum of virtual pair of masses:

$$
m_{3 \mathrm{~A}}+m_{3 \mathrm{~B}}=m_{3}
$$


- Static moments are equal:

$$
\begin{gathered}
m_{3 \mathrm{~A}} l_{a}=m_{3 \mathrm{~B}} l_{b} \\
m_{3 \mathrm{~A}} l_{a}^{2}+m_{3 \mathrm{~B}} l_{b}^{2}=I_{C G 3}
\end{gathered}
$$

Subsequently we obtain the approximate dynamic model of the coupler, and finally the equivalent masses from the following equations:

$$
\begin{aligned}
m_{3 \mathrm{~A}} & =m_{3} \frac{l_{\mathrm{B}}}{l_{\mathrm{A}}+l_{\mathrm{B}}} \\
m_{3 \mathrm{~B}} & =m_{3} \frac{l_{\mathrm{A}}}{l_{\mathrm{A}}+l_{\mathrm{B}}} \\
l_{a} & =\frac{I_{C G 3}}{m_{3}+l_{\mathrm{B}}}
\end{aligned}
$$

This simplification enables to obtain the exact dynamic model of the mechanism (Fig. 7).

Two masses are concentrated in the joint connection $\mathrm{A}$, equivalent crank mass $m_{2 \mathrm{~A}}$ and equivalent coupler mass $m_{3 \mathrm{~A}}$ [12]. Sum of the two masses represents the overall mass at point A:

$$
m_{\mathrm{A}}=m_{2 \mathrm{~A}}+m_{3 \mathrm{~A}}
$$

Also, two masses are concentrated at point $B$, equivalent coupler mass $m_{3 B}$ and the equivalent slider mass $m_{4 \mathrm{~B}}$. Sum of these two masses represents the overall mass at point B:

$$
m_{\mathrm{B}}=m_{3 \mathrm{~B}}+m_{4 \mathrm{~B}}
$$

Now the model consists of two masses which perform either full rotation $\left(m_{\mathrm{A}}\right)$ or full translation $\left(m_{\mathrm{B}}\right)$, which simplifies the dynamic considerations.

\section{FORCE ANALYSIS OF THE CM}

Force graphs of the crank, slider, coupler and supports are based on respective force Balance equations.

Subscript $i$ denotes inertial forces, subscript 4B force due to slider mass, subscript 3B force due to coupler mass at point $\mathrm{B}$, subscript $3 \mathrm{~A}$ force due to coupler mass at point $\mathrm{A}$, subscript $2 \mathrm{~A}$ force due to crank mass at point $\mathrm{A}$.

Overall force between slider and guide $F_{S G}$ :

$$
\vec{F}_{S G}=\vec{F}_{i_{S G 4 \mathrm{~B}}}+\vec{F}_{i_{S G 3 \mathrm{~B}}}=-\left(m_{4}+m_{3 \mathrm{~B}}\right) a_{\mathrm{B}} \tan \phi \vec{\jmath}
$$

Overall force at joint B:

$$
\vec{F}_{34}=\vec{F}_{i 34-4 \mathrm{~B}}+\vec{F}_{i 34-3 \mathrm{~B}}=m_{4} a_{\mathrm{B}} \vec{\imath}-\left(m_{4}+m_{3 \mathrm{~B}}\right) a_{\mathrm{B}} \tan \phi \vec{\jmath}
$$

Overall force at joint A: $\vec{F}_{32}=\vec{F}_{i 32-3 \mathrm{~A}}+\vec{F}_{i 32-4 \mathrm{~B}}+\vec{F}_{i 32-3 \mathrm{~B}}=$

$$
\left[m_{3 \mathrm{~A}} r \omega^{2} \cos \omega t-\left(m_{3 \mathrm{~B}}+m_{4}\right) a_{\mathrm{B}}\right] \vec{\imath}+\left[\left(m_{3 \mathrm{~A}} r \omega^{2} \sin \omega t\right)+\left(m_{3 \mathrm{~B}}+m_{4}\right) a_{\mathrm{B}} \tan \phi\right] \vec{\jmath}
$$

Overall force at support $\mathrm{O}$ :

$$
\begin{gathered}
\vec{F}_{02}=\vec{F}_{32-\mathrm{A}}+\vec{F}_{i 02}=\left[\left(m_{2 \mathrm{~A}}+m_{3 \mathrm{~A}}\right) r \omega^{2} \cos \omega t+\left(m_{3 \mathrm{~B}}+m_{4}\right) a_{\mathrm{B}}\right] \vec{\imath}+ \\
+\left[\left(m_{2 \mathrm{~A}}+m_{3 \mathrm{~A}}\right) r \omega^{2} \sin \omega t+\left(m_{3 \mathrm{~B}}+m_{4}\right) a_{\mathrm{B}} \tan \phi\right] \vec{\jmath}
\end{gathered}
$$

The set of eqs. (16)-(19) describes dynamic behaviour of the slider crank mechanisms and basically is deployed for the simulation of forces generated in the system and needed to be balanced. Though the shaking effect of a slider-crank mechanism in motion is a more complex phenomena, involving not only fundamental, but higher order harmonic components $(2 \omega, 4 \omega, \ldots)$, there is a bundle of very solid reasons why we are going to neglect this aspect: 
1. disturbing effect of higher order components become influential with higher speeds; at the rates of ORBITSHAKER operation it is of a minor importance,

2. focus of the ORBITSHAKER balancing is kept on the first order forces, in order to prevent outboard effects and preserve inboard shaking as much as possible in its natural complexity,

3. concept of CCM in conjunction with a widely variable payload range introduce a series of unknowns into ORBITSHAKER dynamics composition.

Therefore the dynamic model of ORBITSHAKER is kept as simple as possible.

\section{BALANCING OF THE CM}

In the former section 6 the forces at all joints and supports of the crank mechanism are defined. Forces $F_{23}$ and $F_{34}$ are internal forces of the system and do not affect directly the bearing reactions [13]. For the overall quality of mechanism operation and device itself, supporting reactions $F_{21}$ and $F_{41}$ are of the essential importance:

$$
m_{\mathrm{A}} r_{\mathrm{A}}=m_{B A L} r_{B A L}
$$

Considering the eqs. (16) and (17) we conclude that there are two forces at the support $\mathrm{O}$; the first one due to the crank unbalance $\left(m_{2 \mathrm{~A}}+m_{3 \mathrm{~A}}\right)$, and the second, due to the translation of the mass concentrated at the point B, $m_{3 \mathrm{~B}}+m_{4}$, (Fig. 8).

Balancing mass $m_{B A L}$, introduced into the model, compensates the inertial forces induced by the rotation of the mass $m_{\mathrm{A}}$. The sum of forces being transferred to the bearing is:

$$
\vec{F}_{02}+\vec{F}_{S G}=\left(m_{3 \mathrm{~B}}+m_{4}\right) a_{\mathrm{B}} \vec{\imath}
$$

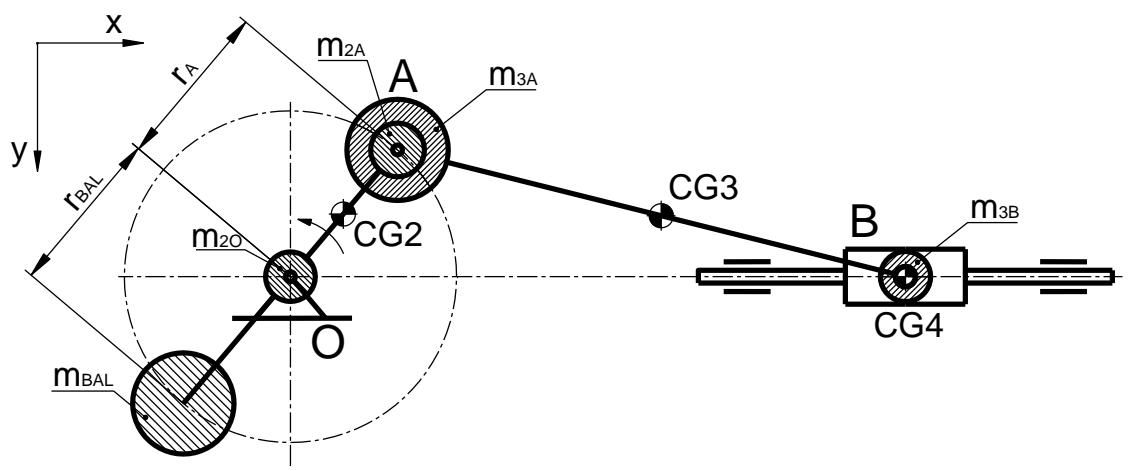

Figure 8: Crank balancing.

By placing an additional or overbalance mass $m_{p}$ (Fig. 9), the state of the ideal balance of the crank rod deteriorates, appears a $y$-component, but the $x$-component decreases, hence decreases the overall resultant force.

It is obvious that such a balancing approach cannot compensate entirely the forces induced by the translation of the mass $m_{B}$ along x-axis. For the ultimate compensation of the active forces it would be necessary to add the oscillating mass with the counter-phase in respect to the slider.

In the case of the ideally balanced crank rod, y-component of the resultant force would be entirely compensated, but the x-component, and hence the resultant force, would remain in a significant extent.

There is no exact formula to define the overbalance mass $m_{p}$, but the suggestion is that it should be within the following range:

$$
\frac{1}{2} m_{\mathrm{B}}<m_{p}<\frac{2}{3} m_{\mathrm{B}}
$$




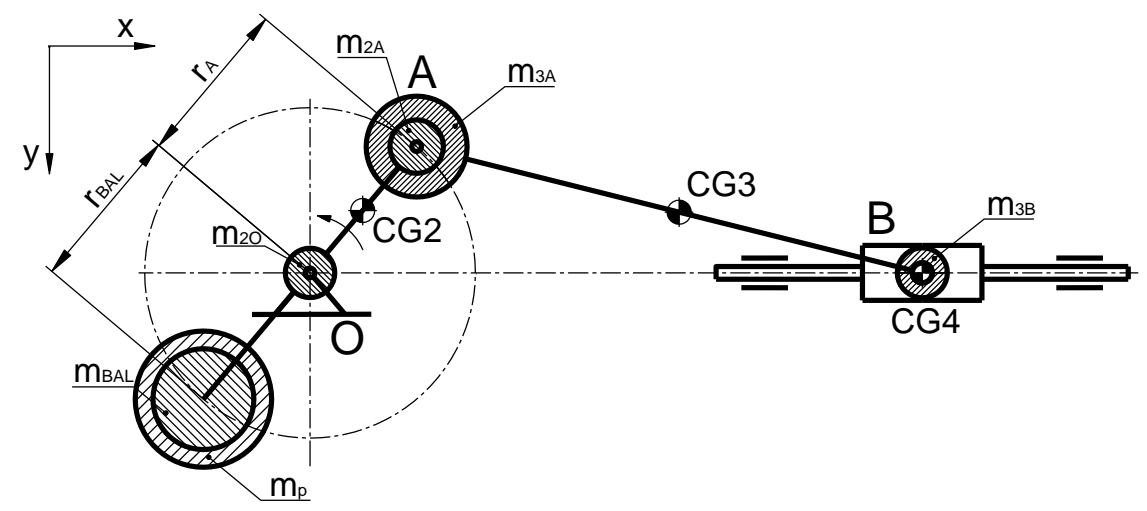

Figure 9: Crank overbalanced.

Orbit shaker is driven by two coupled crank mechanisms CM1 and CM2 (Fig. 5). The main axes are mutually perpendicular.

Stroke of the CM1 is two times greater than the stroke of the CM2, $\left(H^{\mathrm{CM} 1}=60 \mathrm{~mm}\right.$; $\left.H^{\mathrm{CM} 2}=30 \mathrm{~mm}\right)$, while the CM1 angular speed is half of a CM2 speed $\left(n^{\mathrm{CM} 1}=60 \mathrm{RPM}\right.$; $\left.n^{\mathrm{CM} 2}=120 \mathrm{RPM}\right)$.

In order to perform the balancing of two coupled crank mechanisms, it's necessary to balance the CM2 first, and then the CM1. The reason for that lies in the fact that the overall mass of CM2, along with its counter weight is still unknown, at the beginning of the optimization process [14].

\subsection{CM2 balancing}

CM2 crank: $m_{2}=597 \mathrm{~g} ; r_{G 2}=2.9 \mathrm{~mm} ; r=15 \mathrm{~mm} ; m_{2 \mathrm{~A}}=m_{2} \frac{r_{G 2}}{r}=115 \mathrm{~g}$

Coupler: $m_{3}=676 \mathrm{~g} ; l_{A}=l_{B}=99 \mathrm{~mm} ; m_{3 \mathrm{~A}}=338 \mathrm{~g} ; m_{3 \mathrm{~B}}=338 \mathrm{~g}$

Slider: $m_{4}=94227 \mathrm{~g}$

Overall rotating mass at the point $\mathrm{A}$ is $m_{\mathrm{A}}=m_{2 \mathrm{~A}}+m_{3 \mathrm{~A}}=453 \mathrm{~g}$, which means the true balancing of the rotating masses needs $m_{B A L}=453 \mathrm{~g}$, at the correction radius of $r=15 \mathrm{~mm}$, with the magnitude of unbalance $U=m r=6795 \mathrm{~g} \mathrm{~mm}$. Overall translational mass at point B is $m_{\mathrm{B}}=m_{3 \mathrm{~B}}+m_{4}=94565 \mathrm{~g}$.

In accordance with the recommendation from (18), mass should be set within the following range:

$$
47282 \mathrm{~g}<\bar{m}_{O V B A L}^{\mathrm{CM} 2}<63043 \mathrm{~g}
$$

at the radius $r^{\mathrm{CM} 2}=15 \mathrm{~mm}$, with the unbalance $U_{O V B A L}=m_{O V B A L} r$

$$
709237 \mathrm{~g} \mathrm{~mm}<U_{O V B A L}^{\mathrm{CM} 2}<945650 \mathrm{~g} \mathrm{~mm} \text {. }
$$

Apparently the rotating masses are negligible in comparison with the mass of the slider $m_{4}$, therefore the balancing of the whole mechanism can be focused on the balancing of the translational mass at the point $\mathrm{B}$.

Since its unbalance is a significant one, it's necessary to increase the correction radius in order to have the counter weight within the reasonable range. When the correction radius is increased to $r=120 \mathrm{~mm}$, which is applicable by the technical constrains, the counterweight becomes:

$$
5910 \mathrm{~g}<m_{O V B A L}^{\mathrm{CM} 2}<7880 \mathrm{~g}
$$

\subsection{CM1 balancing}

CM1 crank: $m_{2}=665 \mathrm{~g} ; r_{G 2}=5 \mathrm{~mm} ; r=30 \mathrm{~mm} ; m_{2 \mathrm{~A}}=m_{2} \frac{r_{G 2}}{r}=111 \mathrm{~g}$

Coupler: $m_{3}=676 \mathrm{~g} ; l_{A}=l_{B}=99 \mathrm{~mm} ; m_{3 \mathrm{~A}}=338 \mathrm{~g} ; m_{3 \mathrm{~B}}=338 \mathrm{~g}$ 
The overall slider mass of the CM1, involves the gross mass of the CM2, all along with the mass of the weight $m_{O V B A L}^{\mathrm{CM} 2}$ which is still undefined. In the first iteration, we can assume that the mass is $m_{O V B A L}^{\mathrm{CM} 2}=6900 \mathrm{~g}$, which represents the mid value of the expected range.

In that case overall slider mass is:

$$
m_{4}=143854 \mathrm{~g}
$$

Just the same as with the CM2, the rotating masses are negligible in comparison with the mass of the slider $m_{4}$, therefore the balancing of the whole mechanism can be reduced to balancing of the translationally oscillating mass at point B.

Reflected by the same reasons as the case of CM2 balancing, correction radius should be increased to the highest possible rate, which is $r=143 \mathrm{~mm}$ for the CM1.

In that case the estimated counter weight mass is:

$$
15125 \mathrm{~g}<m_{O V B A L}^{\mathrm{CM} 1}<20166 \mathrm{~g}
$$

\section{MOTION SIMULATION, DETERMINATION OF REACTIVE SUPPORT FORCES AND SELECTION OF OPTIMAL COUNTER WEIGHTS}

Simulation of the coupled crank mechanisms CM1 and CM2 motion has been performed with Solid Works Motion software package [15]. In the phase 1 the reactive forces in the crank mechanism CM2 are measured for the different counter weights, in the span $5.5 \mathrm{~kg}$ to $8.5 \mathrm{~kg}$, with $0.5 \mathrm{~kg}$ steps. Results of the simulation are presented in Table III.

The results show that a minimal reactive force acts with a counter weight mass of $6.5 \mathrm{~kg}$, at related speed of $n=120$ RPM. Optimal counter weight is within a range of recommended, calculated values.

Table III: Support forces of the CM2.

\begin{tabular}{|c|c|c|c|c|c|c|c|}
\hline$m_{O V B A L}^{\mathrm{CM} 2}[\mathrm{~kg}]$ & 5.5 & 6.0 & 6.5 & 7.0 & 7.5 & 8.0 & 8.5 \\
\hline$F_{X}[\mathrm{~N}]$ & 140 & 130 & 119 & 109 & 99 & 89 & 79 \\
\hline$F_{Y}[\mathrm{~N}]$ & 96 & 107 & 117 & 127 & 137 & 147 & 157 \\
\hline$F_{R}[\mathrm{~N}]$ & 140 & 130 & 120 & 127 & 137 & 147 & 157 \\
\hline
\end{tabular}

When an optimal counter weight for the CM2 is selected, the simulation starts with the CM1 motion and a reactive force measurement.

Under the regular conditions CM1 and CM2 operate in a conjunction, and two motions mutually interact. In order to cut the cross effect between them, CM2 is kept motionless.

According to the calculus from the previous section, an optimal counter weight might be in the range $16 \mathrm{~kg}$ to $21 \mathrm{~kg}$.

Results of the motion simulation of the CM1 are presented in Table IV.

It is apparent that the minimal reactive force at the CM1 occurs with the counter weight of $18 \mathrm{~kg}$, at related speed of $n=60 \mathrm{RPM}$.

Table IV: Bearing forces for the CM1 (CM2 is OFF).

\begin{tabular}{|c|c|c|c|c|c|c|c|}
\hline$m_{O V B A L}^{\mathrm{CM} 1}[\mathrm{~kg}]$ & 15 & 16 & 17 & 18 & 19 & 20 & 21 \\
\hline$F_{X}[\mathrm{~N}]$ & 120 & 113 & 107 & 102 & 96 & 89 & 82 \\
\hline$F_{Y}[\mathrm{~N}]$ & 84 & 89 & 95 & 101 & 107 & 113 & 119 \\
\hline$F_{R}[\mathrm{~N}]$ & 121 & 115 & 109 & 102 & 108 & 114 & 120 \\
\hline
\end{tabular}

Next step is the analysis of the simultaneous operation CM1 and CM2. Results of the coupled crank mechanisms operation are presented in Table V. 
Table V: Support forces for the CM1 (CM2 is ON).

\begin{tabular}{|c|c|c|c|c|c|c|}
\hline$m_{O V B A L}^{\mathrm{CM} 1}[\mathrm{~kg}]$ & 16 & 17 & 18 & 19 & 20 & 21 \\
\hline$F_{X}[\mathrm{~N}]$ & 177 & 171 & 165 & 158 & 152 & 146 \\
\hline$F_{Y}[\mathrm{~N}]$ & 104 & 110 & 119 & 123 & 129 & 135 \\
\hline$F_{R}[\mathrm{~N}]$ & 177 & 171 & 165 & 158 & 160 & 165 \\
\hline
\end{tabular}

Finally, it can be concluded that the influence of inertial forces generated by the CM2 operation, varies optimal counter weight for just $1 \mathrm{~kg}$ or $5 \%$, shifting $m_{O V B A L}^{\mathrm{CM} 1}$ from 18 to 19 $\mathrm{kg}$.

Reactive force graphs of the CM1, with a simultaneous operation of CM2 for counter weights $m_{O V B A L}^{\mathrm{CM} 1}=19 \mathrm{~kg}$ and $m_{O V B A L}^{\mathrm{CM} 2}=6.5 \mathrm{~kg}$ with respective angular speed $n^{\mathrm{CM} 1}=60 \mathrm{RPM}$, $n^{\mathrm{CM} 2}=120 \mathrm{RPM}$, are presented in Fig. 10 [16].

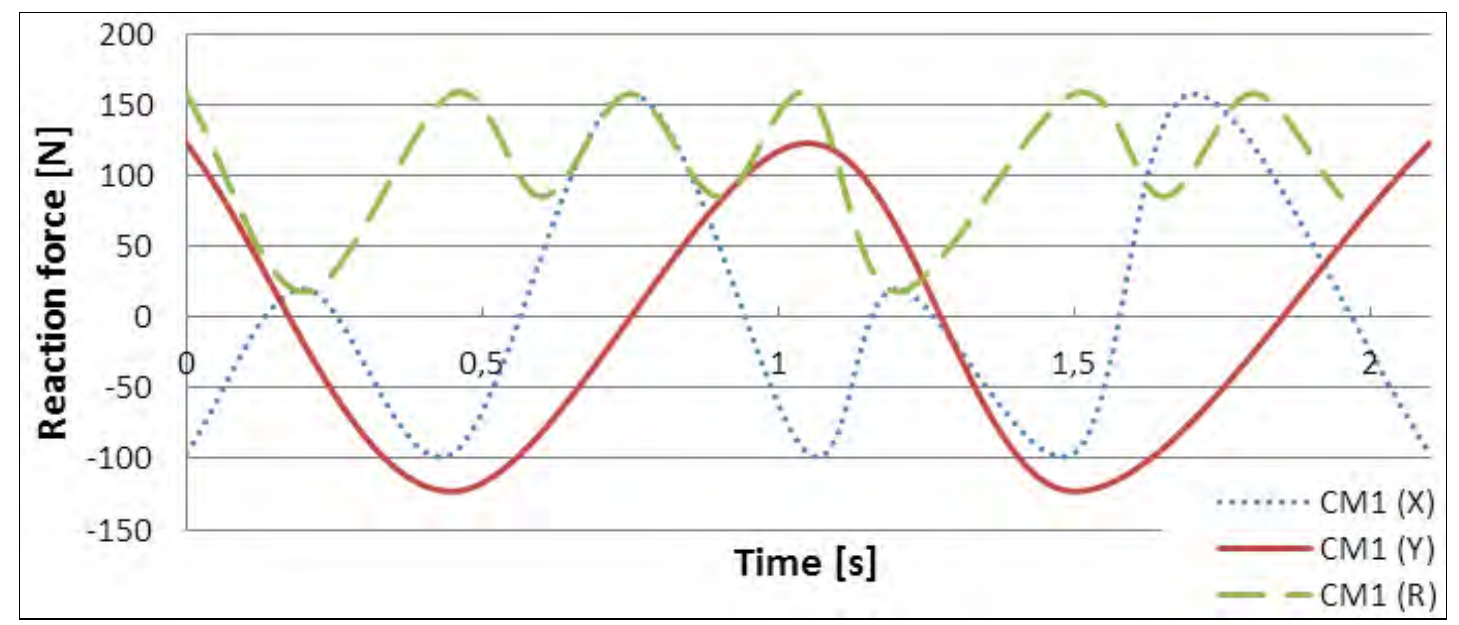

Figure 10: Reaction forces (CM1).

\section{MODEL AND REAL DESIGN}

Parallel to 3D model analysis real assembly definition was conducted. All uncertainties about parts placing, shape design and possible part collision were closely considered through a 3D modelling. Machine assembly was created out of custom modelled parts, as well from standard library. Fig. 11 shows an exploded view of the 3D model. This render includes upper and lower platform, driven respectively by the CM1, and CM2. Also, it shows the position of the electric motor with gearbox that simultaneously drives both mechanisms.

Fig. 12 presents the model of coupled crank mechanisms, with the counterweights.

The smooth manufacturing process proved that a virtual $3 \mathrm{D}$ assembly cancelled all potential design troubles.

\section{CONCLUSION}

The CCM is an orbiting device aimed for the allantoic fluid shaking used in influenza vaccine production. The unique design of the CCM allows simple control of the shaking dynamics. Coupled Crank Mechanisms are simultaneously propelled by single electric motor. Shaking intensity is simply varied by electric motor speed up or slows down. Range of the acceleration magnitude in the extent 10:1 is the result of the speed scaling approximately $3: 1$. 


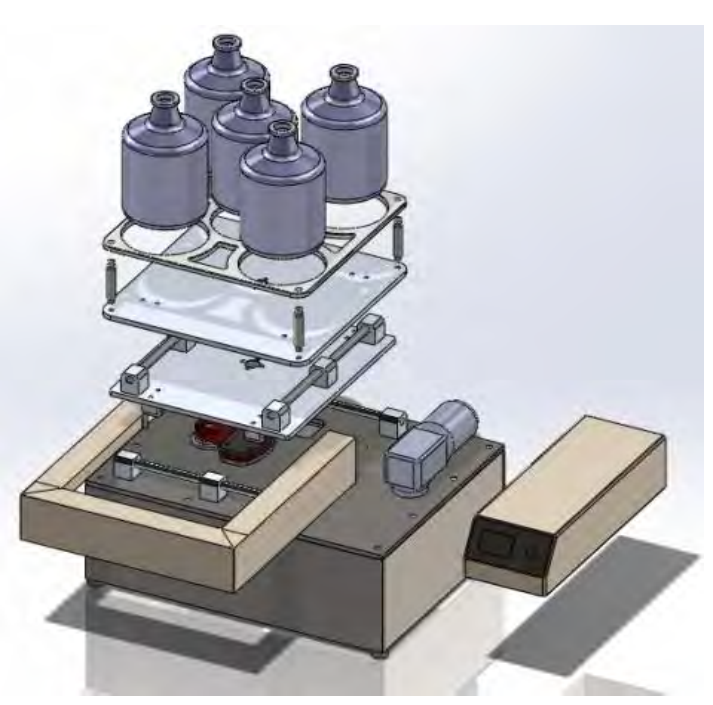

Figure 11: Exploded view of a 3D model.

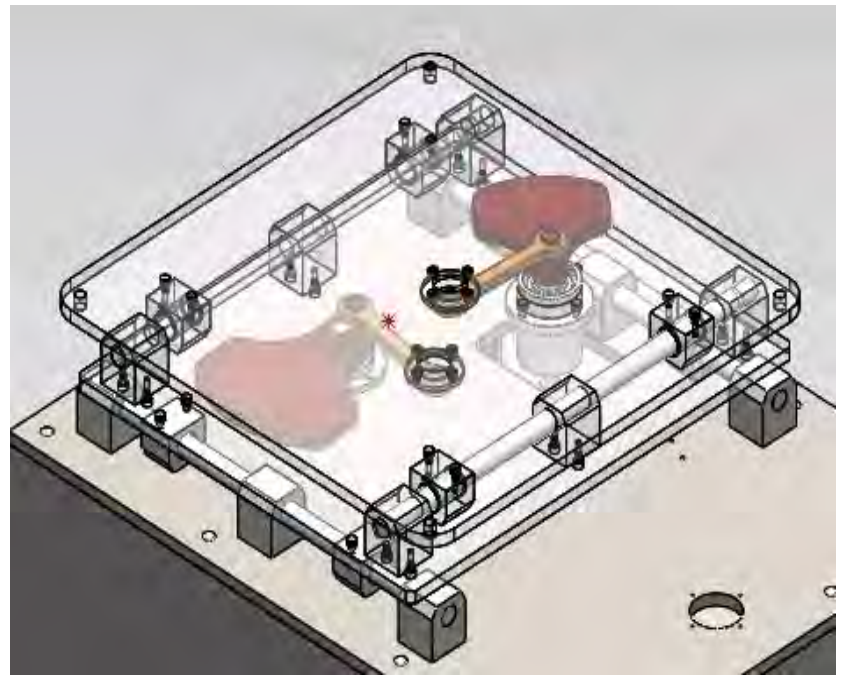

Figure 12: Model of mechanisms CM1 and CM2.

The orbiting motion performed by CCM develops over Planar Figure Eight Orbiting Path. The CCM is a significantly improved concept, compared to the conventional shaking devices for the frequent acceleration shifts over each cycle. The embedded Crank Mechanism in the Orbit Shaker is well-known for its simplicity and durability. Moreover, it is of an easy-tobalance kind. The design of the Orbit Shaker, assisted with the Computer Aided Development was amazingly simple. A series of the design modifications were made rapidly and easily, reducing the hours of engineering work. Apparently there was no need to experiment with a working model. Upon the initial innovative idea the whole research was completed on the computer.

\section{ACKNOWLEDGEMENT}

Authors gratefully acknowledge the financial support from the Ministry of Education, Science and Technological Development of the Republic of Serbia under the projects ON174004 and TR35040.

\section{REFERENCES}

[1] Goldstein, M. A.; Tauraso, N. M. (1970). Effect of formalin, $\beta$-propiolactone, merthiolate and ultraviolet light upon influenza virus infectivity, chicken cell agglutination, hemagglutination, and antigenicity, Applied Microbiology, Vol. 19, No. 2, 290-294

[2] Wright, G. G.; Angelety, L. H. (1971). Effect of the method of agitation on the accumulation of protective antigen in cultures of Bacillus anthracis, Applied Microbiology, Vol. 22, No. 1, 135136

[3] Heidolph Instruments GmbH \& Co.KG. Hei-MIX Shakers \& Mixers, from http://www.heidolphinstruments.com/products/shakers-mixers/, accessed on 15-10-2010

[4] Norton, R. L. (1992). Design of Machinery, $2^{\text {nd }}$ ed., McGraw-Hill, Boston

[5] Madison, J. G. (1996). CNC Machining Handbook, Industrial Press Inc, New York

[6] Hrones, J.; Nelson, G. (1951). Analysis of the four-bar linkage, Wiley, New York

[7] Brigham, E.; DeStefano, C.; Killoy, Z. (2013). Slider - Crank Mechanism for Demonstration and Experimentation MQP, Final Draft, E-project-042513-103948, 93 pages, Worcester Polytechnic Instutute, Worcester

[8] Veg, A.; Šiniković, G.; Veg, E. (2010). Orbiting Mechanism - Computer Aided Development (CADE), Proceedings of The First IFToMM Asian Conference on Mechanism and Machine Science, 636-641 
[9] Marinkovic, Z.; Marinkovic, D.; Petrovic, G.; Milic, P. (2012). Modelling and simulation of dynamic behaviour of electric motor driven mechanisms, Technical Gazette, Vol. 19, No. 4, 717 725

[10] Flores, P. (2011). A methodology for quantifying the kinematic position errors due to manufacturing and assembly tolerances, Strojniski vestnik - Journal of Mechanical Engineering, Vol. 57, No. 6, 457-467, doi:10.5545/sv-jme.2009.159

[11] Mariti, L.; Mucino, V. H.; Pennestri, E.; Cavezza, A.; Gautam, M.; Valentini, P. P. (2014). Optimization of a high-speed deployment slider-crank mechanism: A design charts approach, Journal of Mechanical Design, Vol. 136, No. 7, Paper No: MD-12-1345, 7 pages, doi:10.1115/ 1.4025702

[12] Rao, J. S.; Dukkipati, R. V. (2006). Mechanism and Machine Theory, New Age International Publishers, New Delhi

[13] Rangwala, S. (2006). Reciprocating Machinery Dynamics, New Age International Publishers, New Delhi

[14] Rothbart, H. A. (2006). Mechanical design handbook: measurement, analysis, and control of dynamic systems, McGraw-Hill, Columbus

[15] Kljajin, M.; Pisacic, K.; Kecek, D.; Bolaric, D. (2014). Comparison of kinematic analysis methods at one demonstration mechanism, Technical Gazette, Vol. 21, No. 3, 657-664

[16] Veg, A.; Šiniković, G.; Andrejević, R.; Veg, E. (2011). Computer Aided Balancing (CAB) applied on an orbiting mechanism, Proceedings of the $13^{\text {th }}$ World Congress in Mechanism and Machine Science, A7-613 